\title{
Tamm resonances and minibands in the models of atomic chains and superlattices
}

\author{
O.I.Gerasimov, N.N.Khudyntsev \\ Odesa State Hydrometheorological Institute, \\ 100 Lvivska Str., 270011 Odesa, Ukraine
}

Received June 5, 1998, in final form January 26, 2000

\begin{abstract}
The spectrum of the modelled regular superlattice which includes contacts is investigated using the transfer-matrix formalism. It is shown that the separated pair resonances in the chain spectrum (which are distributed in the permitted and in the forbidden zones, respectively) appear due to the existence of a single contact. The eroding of the noted minizones after making correlations between the separate regular segments in the superlattice is demonstrated. Similar resonances and minizones which can be called the Tamm resonances and minizones are considered to be useful elements for describing the surface states and zone-band structure of real systems within the restricted geometry.
\end{abstract}

Key words: contact, superlattice, minibands, spectrum

PACS: $68.65 .+G, 73.90 . D x$

\section{Introduction}

The modelling of the low-dimensional atom-molecular complexes, adatomic clusters, chains, lattices and superlattices belongs to a number of traditionally and actively researched problems of the solid state theory [1]. The urgency of the research is limited by the essentiality and the difficulties of a direct microscopic description of the recently synthesized low-dimensional atom-molecular complexes and other physical objects with the symmetry, for instance, different from the translational one (icosahedra or quasicrystallic) [2]. This way, specifically, in [3], the one-dimensional Kronig-Penny superlattice with a regular intrinsic structure determined by the combination of zero-radius potentials [4] was studied. Then, the simple analytical model is suggested [1] permitting to explore some general properties of superlattices, separate contacts (interfaces) being the model elements for describing semiconductor and metallic films, quasicrystalls and other objects. Such a model can be used in constructing more general theories. Using the transfer-matrix formalism this paper 
considers the problem regarding the spectrum of a regular superlattice as well as suggests a simple method of describing the contact in the superlattice structure. It is shown that the existence of a single contact causes separate pair resonances in the chain spectrum (which are distributed in the permitted and in the forbidden zones, respectively). The eroding of the mentioned minizones after making a connection between the separate regular segments in the superlattice is demonstrated as well. Similar resonances and minizones can be called the Tamm's ones, being useful elements for describing the surface states and the zone structure of the real low-dimensional systems.

\section{Statement of the problem. Transfer-matrix formalism}

Consider the sequence of nonstructured particles forming a one-dimensional superlattice with the alternation of the regular segments which are characterized by different periods as well as by different power constants. The potential energy of the system is modeled using the point-like interactions:

$$
U(x)=\frac{1}{2} \sum_{n, n^{\prime}=-\infty}^{+\infty}\left[V_{a} \delta(x-n a)+V_{b} \delta\left(x-n^{\prime} b\right)\right],
$$

where $a, b$ are the lattice periods, $V_{a}, V_{b}$ are the power constants of the centrums of different segments. We also assume that the mass (or the effective mass) of the scattered particle and the Plank constant are equal to 1 . The description of one-dimensionality (quasi-onedimensionality) of the zond-particle motion is given, for instance, in [8]. The summation in (1) is carried out over all different indexes $n$ and $n^{\prime}$. Modelling of the superlattices using the potential (1) is demonstrated in figure 1. The con-

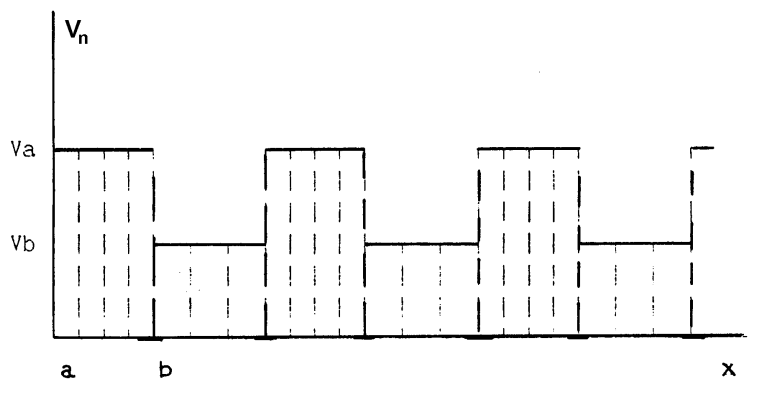

Figure 1. The schematic scene of the superlattice potential with the translational symmetry $(a, b$ are the segments period, $V_{a}, V_{b}$ are the power constants). tacts of segments with different lattice parameters are shown by means of the contrast horizontal lines. For simplicity, the symmetry in the position of superlattice segments is suggested as a translational $(\mathrm{Na}, \mathrm{Nb}=\mathrm{const})$. The refusal from this assumption leads us to the problem of a regular chain with defects, which was considered in [9]. In such a case with a superlattice, a quasiperiod composed of two nearest segments (see figure 1) could be considered.

The Shrödinger equation of the considered problem could be written in the following form:

$$
\left[-\frac{1}{2} \frac{\mathrm{d}^{2}}{\mathrm{~d} x^{2}}+U(x)-E\right] \Psi(x)=0
$$


where $E$ and $\Psi(x)$ are respectively the eigenvalue of the energy and of the wave function of zond-particle in the field of the potential $U(x)$.

For the model potential (1), the zond-particle motion can be considered as a free motion and the wave function can be described by superposition of two plane waves:

$$
\Psi(x)=A \exp (\mathrm{i} k x)+B \exp (-\mathrm{i} k x),
$$

where $k^{2}=2 E$. The coefficients $A$ and $B$ could be extracted from the boundary conditions.

The natural boundary conditions in the point $d$ of $n$-atomic centrum for the wave function and their first derivative could be chosen in the following way:

$$
\Psi(d+0)=\Psi(d-0), \quad \frac{\mathrm{d} \Psi(d+0)}{\mathrm{d} x}=\frac{\mathrm{d} \Psi(d-0)}{\mathrm{d} x}+V_{n} \Psi(d-0) .
$$

The boundary conditions (4) follow from the original structure of equation (2) and also from the general properties of the zero-radius potential (see [4]). In the matrix form, the boundary conditions (4) could be rewritten as:

$$
\widehat{\mathbf{T}}_{n}\left(\begin{array}{l}
A_{n} \\
B_{n}
\end{array}\right)=\left(\begin{array}{c}
A_{n+1} \\
B_{n+1}
\end{array}\right),
$$

where $\widehat{\mathbf{T}}_{n}$ is the transfer-matrix being the square unimodular matrix of the size $2 \times 2$ in the Kelly form [6]:

$$
\widehat{\mathbf{T}}_{n}=\left(\begin{array}{cc}
\alpha_{n}^{*} & \beta_{n}^{*} \\
\beta_{n} & \alpha_{n}
\end{array}\right) .
$$

Within the framework of the transfer-matrix formalism, the problem regarding the spectrum of a one-dimensional system of point-like centrums could be solved exactly. A general approach to using the matrix form for the boundary conditions for their connection with the energy spectrum as well as some characteristics of particle transmission in the field of different potentials is given in [6]. This formalism also makes it possible to determine some transport characteristics for the system studied (for example, the Landauer resistance coefficient [9] which represents the relation between the quantum-mechanical coefficients of transmission and reflection, respectively). Following the general approach described in $[6,9]$, the expressions for the spectrum $E$ and the resistance coefficient $\rho$ could be extracted in the form of the following relations (the conversion from the boundary conditions (4) to the following expressions have also been considered in [9]):

$$
\begin{gathered}
E=\frac{Q^{2}}{2}, \quad \cos Q a=\frac{1}{2} \operatorname{Sp} \widehat{\mathbf{T}}_{n}, \\
\rho=\frac{K_{\text {tran }}}{K_{\text {refl }}}=\left|\beta_{n}\right|^{2},
\end{gathered}
$$

where $Q$ is the quasi-wave vector of the zond-particle, $K_{\text {tran }}, K_{\text {refl }}$ are the reflection and transmission quantum-mechanical coefficients. The expressions for $\alpha_{n}, \beta_{n}$ are given in [9]. 
Two types of a zond-particle motion in the field of the system considered would be analyzed below: the resonance and nonresonance tunnelling [8]. Completely real and completely imaginary values of the quasi-wave vector $Q$ correspond to these cases respectively. Now consider the pure resonance tunnelling (otherwise, we can put $Q \rightarrow \mathrm{i} Q$ to obtain correlations). If $N_{a}, N_{b}>1$, the diagonal representation of transfer-matrix $\widehat{\mathbf{T}}_{n}$ could be written in the following form:

$$
\widehat{\mathbf{T}}_{n d}=\widehat{\mathbf{S}}_{n}^{-1} \widehat{\mathbf{T}}_{n} \widehat{\mathbf{S}}_{n}=\left(\begin{array}{cc}
\lambda_{n+} & 0 \\
0 & \lambda_{n-}
\end{array}\right),
$$

where $\lambda_{n \pm}$ are the eigenvalues of the matrix (6), $\widehat{\mathbf{S}}_{n}=\left(\begin{array}{cc}S_{n+} & S_{n-} \\ 1 & 1\end{array}\right)$ is the diagonalized matrix, $S_{n \pm}=\left(\lambda_{n \pm}-\alpha_{n}\right) \beta_{n}^{-1}$.

For instance, in the case of regular chain with the period $a\left(V_{a}=V_{b}=V, d=n a\right)$ :

$$
\begin{aligned}
& \lambda_{a \pm}=\exp ( \pm \mathrm{i} q a), \quad \cos q a=\cos k a+\frac{V}{k} \sin k a \\
& \alpha_{a}=\left(1+\frac{\mathrm{i} V}{k}\right) \exp (-\mathrm{i} k a), \quad \beta_{a}=\frac{\mathrm{i} V_{a}}{k} \exp (\mathrm{i} k a) .
\end{aligned}
$$

The chain containing isotopic and (or) shift defects was considered in detail in [9]. Within the next chapters, the suggested transfer-matrix formalism will be used for describing the complex superlattice (see figure 1).

\section{A model of contact within the superlattice. The spectrum and resistance of a single contact}

Consider the superlattice within the quasi-period which includes $N_{a}$ atoms in the segment with the period $a$ and $N_{b}$ atoms in the segment with the period $b$. The respective transfer-matrix has the following form:

$$
\widehat{\mathbf{T}}_{c}=\left(\widehat{\mathbf{T}}_{a}\right)^{N_{a}}\left(\widehat{\mathbf{T}}_{b}\right)^{N_{b}}
$$

where $\widehat{\mathbf{T}}_{a}$ and $\widehat{\mathbf{T}}_{b}$ are the transfer-matrix (6) with the elements (10) for the regular chains with periods $a$ and $b$, respectively.

We are interested in the diagonal representation of the matrix $\widehat{\mathbf{T}}_{c}$ (only the numerical solution of the problem could be provided within all other representations). As it was shown in $[6,9]$, the diagonal representation of the matrix (6) with different parameters doesn't exist. Using matrix $\widehat{\mathbf{S}}_{a}$ (as a diagonalizing one), using (9) and (10) we obtain:

$$
\widehat{\mathbf{T}}_{c d}=\widehat{\mathbf{S}}_{a}^{-1} \widehat{\mathbf{T}}_{c} \widehat{\mathbf{S}}_{a}=\left(\widehat{\mathbf{T}}_{a d}\right)^{N_{a}} \widehat{\mathbf{T}}_{q}\left(\widehat{\mathbf{T}}_{b}\right)^{N_{b}} \widehat{\mathbf{T}}_{p}
$$

where $\widehat{\mathbf{T}}_{a, b d}$ are the transfer-matrixes within the diagonal representation, and

$$
\widehat{\mathbf{T}}_{q}=\widehat{\mathbf{S}}_{a}^{-1} \widehat{\mathbf{S}}_{b}, \quad \widehat{\mathbf{T}}_{p}=\widehat{\mathbf{S}}_{b}^{-1} \widehat{\mathbf{S}}_{a}
$$


In this representation, the contact of two regular segments could be considered as the last one, just like the regular chain that includes two defects in their structure could be described by the non-diagonal transfer-matrixes $\widehat{\mathbf{T}}_{q}$ and $\widehat{\mathbf{T}}_{p}$ (where matrix $\widehat{\mathbf{T}}_{p}$ characterizes the next contact from the left to the right). Thus, the transfermatrix which corresponds to a single contact is $\widehat{\mathbf{T}}_{q}$, namely:

$$
\widehat{\mathbf{T}}_{q}=\frac{1}{\lambda_{a+}-\lambda_{a-}}\left(\begin{array}{cc}
\lambda_{b+}-\lambda_{a-}+\alpha_{a}-\alpha_{b} & \lambda_{b-}-\lambda_{a-}+\alpha_{a}-\alpha_{b} \\
\lambda_{a+}-\lambda_{b+}+\alpha_{b}-\alpha_{a} & \lambda_{a+}-\lambda_{b-}+\alpha_{b}-\alpha_{a}
\end{array}\right) .
$$

Note that the expression for the matrix $\widehat{\mathbf{T}}_{p}$ could be obtained from (14) by changing the periods $a$ and $b$. Finally, for describing the zone structure of the segment which includes the contact we obtain:

$$
E_{c}=\frac{Q_{c}^{2}}{2}, \quad \cos Q_{c} a=1+\frac{\lambda_{b+}-\lambda_{b-}}{\lambda_{a+}-\lambda_{a-}},
$$

where $Q_{c}$ is the quasi-wave vector of zond-particle in the field of the segment with the contact.

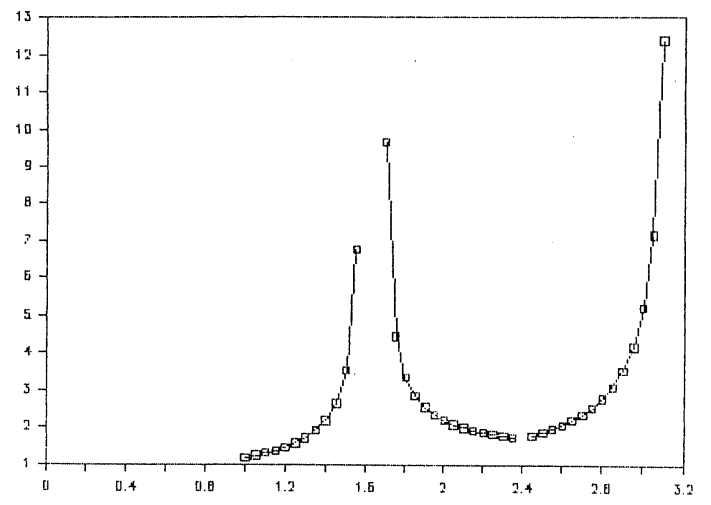

Figure 2. The dependence of $\operatorname{Sp} T_{q}$ (vertical axis) from the wave vector $k a$ (horizontal axis): $V_{a}=0.4, b / a=4$.

The results of the numerical calculations using the formulas (15) are presented in figure 2 . We should take into account the deformation of the zone structure of the lattice consisting of two regular segments in comparison with the structures of zones of separate regular segments. The positions of a boundary limit of the permitted and of the forbidden zones are shifted, and the separate resonances (interfaces) have appeared which correspond to the forbidden levels in the permitted zones and (or) to the permitted levels in the forbidden zones, respectively. Those resonances (which have a Tamm's nature) are split into pairs near the point of the zone boundary $q=\pi n / a$ ( $n$ is the integer number). Note that the same effect of splitting the separate resonances was also found in the spectrum of the regular chains that include the isotopic- and (or) shift-type defects [11].

Now consider the resistance coefficient (see chapter 2) which is limited by the contact of two regular segments. Using the substitution of a nondiagonal element of the transfer-matrix (14) into (8) one can obtain:

$$
\rho_{c}=\frac{1}{\sin ^{2} q a}\left[\sin ^{2} \omega+\left(1+\frac{W^{2}}{k^{2}}\right) \sin ^{2} \kappa+2 \sin \kappa \sin \omega\left(\cos \varphi+\frac{W}{k} \sin \omega\right)\right],
$$

where

$$
\omega=q(a-b) / 2, \varphi=(q+k)(a+b) / 2, \kappa=k(a-b) / 2, W=2 V_{a} V_{b}\left(V_{a}+V_{b}\right)^{-1} .
$$



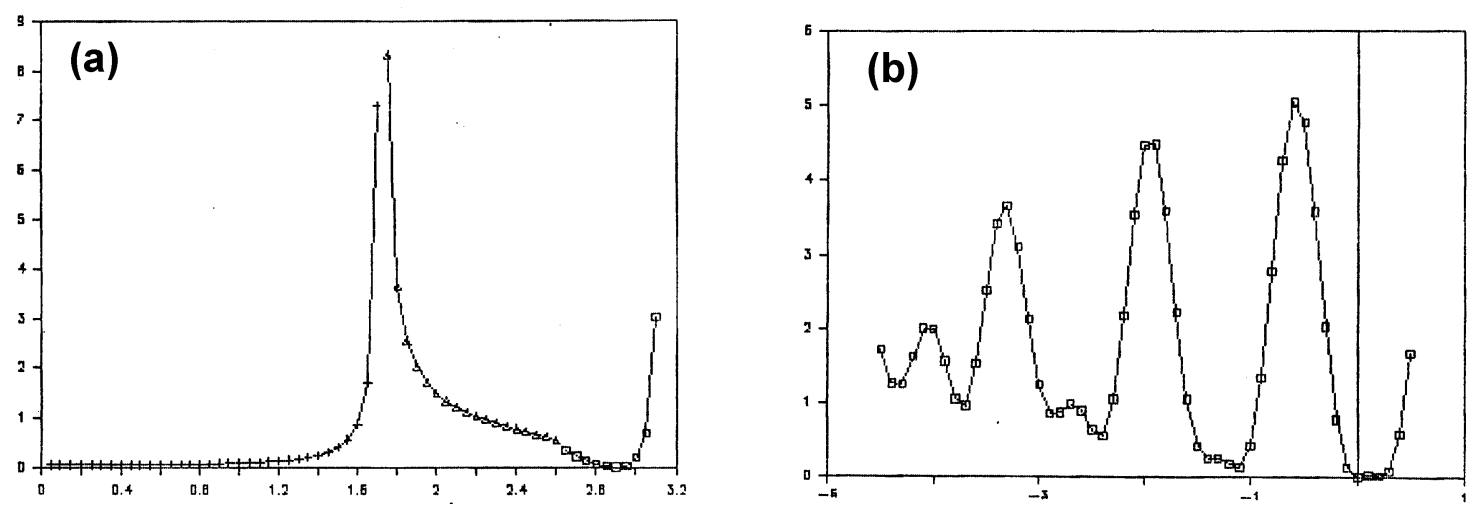

Figure 3. Coefficient of resistivity $\rho_{\mathrm{c}}$ (vertical axis) as a function of $k a$ (a) and of $1-b / a$ (b) (horizontal axis): $V_{a}=2, k a=2.45$.

The coefficient $\rho_{c}$ as a function of $k a$ and $1-b / a$ which was calculated using formula (16) is shown in figures 3a,b. Besides the simplicity of calculation we put $V_{a}=V_{b}$. The coordinate dependence for the resistance coefficient is not present in the homogeneous chain model. Asimptotically, the resistance coefficient increases near the edges of the zones as a function of the parameter $1-b / a$. Note that at the determined values of $V$ and $b / a$, the decrease of the resistance coefficient up to zero could be observed which corresponds to the regime of zond-particle pure tunnelling (without dissipation). The mentioned properties of the contact can be considered as general properties for any one-dimensional structure having an arbitrary superlattice symmetry.

\section{Spectrum of the superlattice having a translational symme- try}

As it was stressed in chapter 1, the problem of determining the spectrum of the superlattice having a translational symmetry reduces to the similar problem of a regular chain with the change of the chain periods to the superlattice quasi-one scale. Within the framework of the transfer-matrix formalism, we need to calculate the trace of the matrix (11). Finding the solutions of the equation

$$
\left|\widehat{\mathbf{T}}_{c}-\Lambda \widehat{\mathbf{I}}\right|=0
$$

where $\Lambda$ is the eigenvalue of the matrix $\widehat{\mathbf{T}}_{c}, \widehat{\mathbf{I}}$ is the unity matrix, in accordance with (7) the expression for superlattice spectrum could be expressed in the following form:

$$
\begin{gathered}
E_{\mathrm{s}}=\frac{Q_{\mathrm{s}}^{2}}{2} \\
\cos Q_{\mathrm{s}} a=\frac{\left(S_{b+}-S_{a-}\right)\left(S_{a+}-S_{b-}\right) G_{+}+\left(S_{b+}-S_{a+}\right)\left(S_{b-}-S_{a-}\right) G_{-}}{\left(S_{a+}-S_{a-}\right)\left(S_{b+}-S_{b-}\right)},
\end{gathered}
$$

where $G_{ \pm}=\cos q\left(N_{a} a \pm N_{b} b\right)$. 


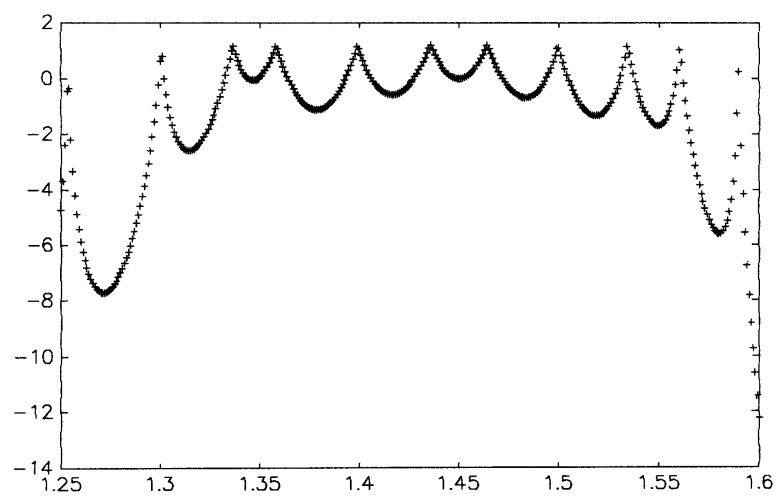

Figure 4. The energy spectrum $E_{\mathrm{s}}$ of the superlattice as a function of $k a$.

The respective pictures for the spectrum (obtained by calculations using the expression (18)) are shown in figure 4 . The complex character of (18) is demonstrated at the determined values of the potential and the lattice period parameters. Taking into account the correlation between separate interfaces, the multiplication of resonance factors of the kind $(\sin q a \sin q b)^{-1}$ occurs. This effect can be interpreted as the erosion of splitting Tamm resonances (interface states) in some minizones.

In the case of disturbance of translational symmetry of the superlattice by the "point-like" defects, the change of zone structure and Tamm minizones would be provided. Thus the considered model gives the exact solution to the problem of calculating the spectrum and introduced transport characteristics of one-dimensional superlattices (including contacts). In spite of their model character, the obtained results should be taken into account for describing the real physical objects having the structure similar to atomic chains and superlattices [10-12].

\section{References}

1. Esaki L. (ed.) Highlights in Condensed Matter Physics and Future Prospects. // NATO ASI Series B, 1991, vol. 285, p. 1-390.

2. Levitov L.S., Riner G. Icosahedral symmetry of quasicrystals. // Letters to JETP, 1988, vol. 47, No. 12, p. 658-667 (in Russian).

3. Tikhodeev S.G. Tamm minizones in the theory of superlattices. // JETP, 1991, vol. 99, No. 6, p. 1871-1880 (in Russian).

4. Demkov Yu.N., Ostrovsky V.N. Method of Zero-radius Ranged Potentials in Physics. Leningrad, Pub. Leningrad State University, 1975 (in Russian).

5. Kelly M.J., Weisbush C. (ed.) The Physics and Fabrication of Microstructures and Microdevices. Berlin, Spr. Verlag, 1986.

6. Ziman J.M. Models of Disorder. The Theoretical Physics of Homogeneously Disordered Systems. Cambrige, Cambrige University Press, 1979.

7. Tamm I.Ye. About the question of surface resonances in the theory of solid bodies zone structure. // JETP, 1933, vol. 3, p. 34-49 (in Russian).

8. Lifshits I.L., Kirpichenkov V.Ya. About the tunnel effects in the disordered systems. - In: Physics of Real Crystals and Disordered Systems. Moscow, Nauka Pub., 1987, p. 272-300 (in Russian).

9. Gerasimov O.I., Zagorodny A.G., Khudyntsev N.N. Structural and electronic properties of atomic chains. Preprint of the Institute for Theoretical Physics, ITP-91-95, Kyiv, 1991. 
10. Gerasimov O.I., Khudyntsev N.N. Tamm's resonances and minibands within the model one-dimensional potential. - In: Thes. of Workshop on Cond.Matt.Phys., Lviv, 1998, p. 69.

11. Gerasimov O.I., Alexeev A.E., Idomsky V.M., Khudyntsev N.N. Conductance properties of dry granular systems within restricted geometry. - In: Thes. of XX IUPAP International conference on statistical physics, Paris, 1998, p. 13.

12. Gerasimov O.I., Alexeev A.E., Idomsky V.M., Khudyntsev N.N. Conductance properties of dry granular systems within restricted geometry. - In: Thes. of NATO ASI, Leiden, 1998.

\title{
Таммівські резонанси та мінізони для модельного одновимірного потенціалу
}

\author{
Герасимов О.І., Худинцев М.М.
}

Одеський державний гідрометеорологічний інститут, 270011 Одеса, вул. Львівська, 100

Отримано 5 червня 1998 р., в остаточному вигляді - 26 січня 2000 p.

У роботі за допомогою формалізму трансфер-матриці розв'язана проблема обчислення спектра регулярної супергратки та запропоновано досить простий метод опису контакту в їі структурі. Показано, що існування одного контакту призводить до виникнення відокремлених парних резонансів у спектрі ланцюжка, а також продемонстровано розмиття таких резонансів у відповідні мінізони після поєднання окремих регулярних сегментів у супергратку. Означені резонанси та мінізони $€$ таммівськими та можуть виступати зручними елементами опису поверхневих станів та зонної структури реальних низьковимірних систем.

Ключові слова: контакт, супергратка, мінізона, спектр

PACS: $68.65 .+G, 73.90 D x$ 